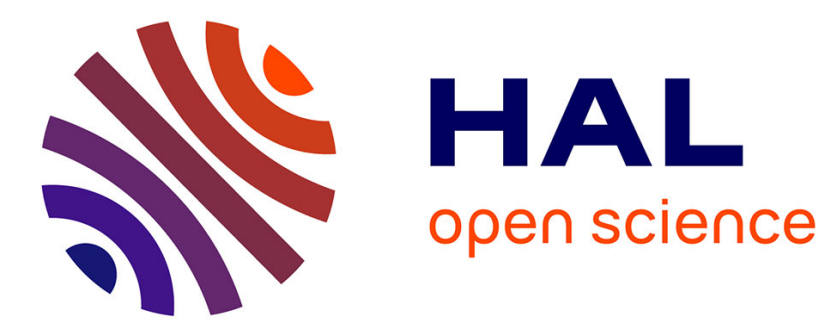

\title{
Interfacial Instability during Granular Erosion
}

Gautier Lefebvre, Aymeric Merceron, Pierre Jop

\section{To cite this version:}

Gautier Lefebvre, Aymeric Merceron, Pierre Jop. Interfacial Instability during Granular Erosion. Physical Review Letters, 2016, 116 (6), pp.068002 10.1103/PhysRevLett.116.068002 . hal-01274966

\section{HAL Id: hal-01274966 https://hal.science/hal-01274966}

Submitted on 16 Feb 2016

HAL is a multi-disciplinary open access archive for the deposit and dissemination of scientific research documents, whether they are published or not. The documents may come from teaching and research institutions in France or abroad, or from public or private research centers.
L'archive ouverte pluridisciplinaire HAL, est destinée au dépôt et à la diffusion de documents scientifiques de niveau recherche, publiés ou non, émanant des établissements d'enseignement et de recherche français ou étrangers, des laboratoires publics ou privés. 


\title{
Interfacial Instability during Granular Erosion
}

\author{
Gautier Lefebvre, Aymeric Merceron, and Pierre Jop* \\ Surface du Verre et Interfaces, UMR 125 CNRS/Saint-Gobain, \\ 39, quai Lucien Lefranc, F-93303 Aubervilliers, Cedex, France
}

(Dated: September 24, 2015)

\begin{abstract}
The complex interplay between the topography and the erosion and deposition phenomena is a key feature to model granular flows such as landslides. Here, we investigated the instability that develops during the erosion of a wet granular pile by a dry dense granular flow. The morphology and the propagation of the generated steps are analyzed in relation to the specific erosion mechanism. The selected flowing angle of the confined flow on a dry heap appears to play an important role both in the final state of the experiment, and for the shape of the structures. We show that the development of the instability is governed by the inertia of the flow through the Froude number. We model this instability and predict growth rates that are in agreement with the experiment results.
\end{abstract}

Understanding sediment transport in Nature is essential to model landscape evolution, such as the transport processes in rivers and their formation [1,2]. Patterns are known to spontaneously develop at the bottom of the river bed depending on the coupling between the flow and the bed geometry through the erosion/transport laws $[3,4]$. Granular flows such as landslides can also alter the underlying ground. However, contrary to the fluid case, the erosion mechanisms are less clear when the granular material is dense. Thus recent studies have focused on the evolution of the interface between an erodible layer and a granular flow [5-7], especially to model the entrainment rates, the velocities and the runout distances. Understanding such processes can also give insights on past and present climates for example [8-10]. Moreover, although the instabilities of riverbeds have been extensively characterized [11], the studies of instabilities in granular flows were generally focused on the flowing layer, thus describing avalanche fronts, roll waves, upward traveling waves [12-15], providing a better understanding of granular flows. As a result, little attention has been paid to the evolution of a granular bed under a solid mechanical load $[16,17]$. Finally, while previous studies on the erosion rate of a cohesive media by a granular flow focused on the physical properties of the cohesive materials (liquid bridges, tensile strength, elastic modulus) [7, 18, 19], the coupling between the erosion mechanism and the interface morphology with the granular flow is still an open question.

In this Letter, we explore the coupling between a flow of dry granular material and a cohesive granular bed. Our experiment exhibits an instability, as a train of steps appears along the initially flat interface. Although this instability shares similarities with the erosion of a dense cohesive bed by fluid flow [3], we show that this system presents unique features linked to the specific properties of granular matter. Using a granular rheology and an erosion law, we can model the phenomenon. The threshold and growth rates of the initial instabilities are in good agreement with the theory, providing insights into the erosion mechanism itself.
We performed experiments in a quasi-two-dimensional cell made of parallel vertical glass plates, whose gap width $W$ was set at 6 and $12 \mathrm{~mm}$. The same polydisperse glass beads of diameter 200-400 $\mu \mathrm{m}$ were used for both the cohesive granular bed and the flow. The cohesive material was obtained by mixing a small quantity of water with the beads to obtain a low water content of $1 \% \mathrm{wt}$, which corresponds to the formation of individual capillary bridges [20]. This material was introduced and packed into the cell to form a heap with a planar free-surface of length ranging from 30 to $35 \mathrm{~cm}$. We injected the dry granular material at the top left of the cell so a flowing layer would from above the wet heap (Fig. 1a). The two remaining parameters are the initial inclination of the heap $\theta_{i}$ and the constant injected flow rate $Q$. Both parameters influence the flow properties, mainly the stresses at the interface $\tau_{b}$ and the mean velocity $u$.

As shown by the time evolution of the heap in figure 1a, erosion occurs at the interface between the cohesive pile and the granular flow, without deposition. Wet beads are slowly extracted by the action of the dry flow. Thus, the volume of the cohesive heap is gradually reduced during the experiment until it reaches a steady state in which the interface is flat and the dry granular material flows without eroding the wet bed. During this erosion process, two different interface morphologies can be observed. Either the eroded interface remains initially flat and parallel to the initial slope, or we observe the creation of step-shaped structures for rather high inclinations and high flow rates. These steps grow and propagate upstream, as shown in figures $1 \mathrm{a}, \mathrm{b}$, then disappear one after the other at the top of the channel. Once they all have disappeared, the erosion stops. In both cases, the final interface is flat and forms an angle $\theta_{f}$ with the horizontal with $\theta_{f}<\theta_{i}$.

Figure $1 \mathrm{~b}$ shows the evolution of the heap free surface with time in an unstable case. The first minutes of the development of the instability see the creation of many structures of small sizes, rapidly merged by a coarseninglike process. The surviving steps go through a growth phase lasting roughly 30 minutes, until they reach a stable shape (height and length): The back of the steps is 

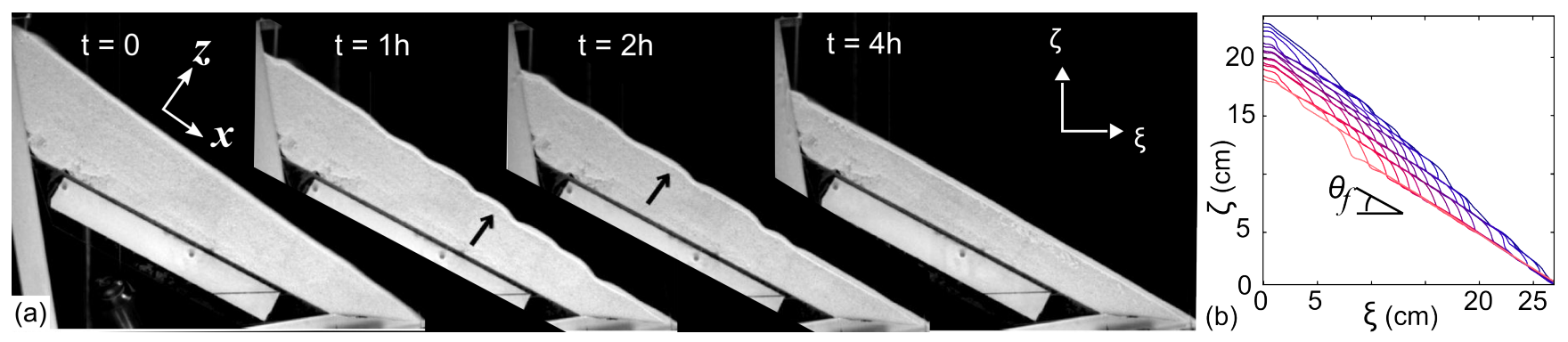

FIG. 1. (a) Successive lateral views of a typical experiment. The shallow flowing granular layer appears brighter above the wet heap. The first frame is the initial state, and the others are snapshots taken during the erosion. The black arrow follows one step. The flow rate is $3.3 \mathrm{~g} / \mathrm{s}$ and the initial inclination $35.8^{\circ}$. The width of one picture corresponds to $27 \mathrm{~cm}$. (b) Successive profiles of the interface, from dark blue to light red, every 15 minutes in the $6 \mathrm{~mm}$ channel, showing the steady propagation of steps over an initial slope angle of $39.4^{\circ}$.

not eroded, and appears as straight parallel lines while the front propagates upwards at a constant speed. We note that, close to the entrance and exit of the channel, the steps are influenced either by the injection point or by the accelerating flow. A single experiment may produce steps with different sizes, even once they are well established. However all the steps display a similar steep front, and a flat back with a well defined slope. Figure 2a shows the shapes of four different steps obtained for the same flow rate and different initial slopes. The front is well fitted by a parabola, whereas the back forms an angle $\theta_{f}$ with the horizontal, which is equal to the final erosion angle. This means that the final equilibrium state is quickly reached locally, i.e. at the back of the steps. Moreover, the quantitative fitting of the parabola corresponds to a free fall under the gravity with an initial velocity of $22 \mathrm{~cm} \cdot \mathrm{s}^{-1}$ (solid red lines) which is comparable to the mean velocity $u$ of the flow on the final slope $\left(11.6 \mathrm{~cm} . \mathrm{s}^{-1}\right)$. This collapse of different step profiles also shows that the shape of the higher fronts are a simple continuation of the smaller ones (fig 2a). We interpret this similar shape as a consequence of the take-off of the granular flow occurring on the front of the steps. As the grains are almost free-falling after this point, there is no feedback from the lower part of the chute toward the upper part of the front of the steps through the dilute flow. This decoupling explains why the shape is kept, regardless of its size. It is worth noting that the erosion mechanism by this dilute flow is also probably different from the one initially occurring with the dense flow. This morphology and this propagation share similarities with the head-cut [21] or step and pool formation in riverbeds [3].

In the following, we focus on the final slope observed for both types of erosion morphology in this system. First let us comment on the stress evolution at the interface. For experiments driven at constant flow rate as these ones, one easily shows that the shear stress is increasing when the slope decreases using granular scaling laws (a result valid along an inclined plane [22], in presence of
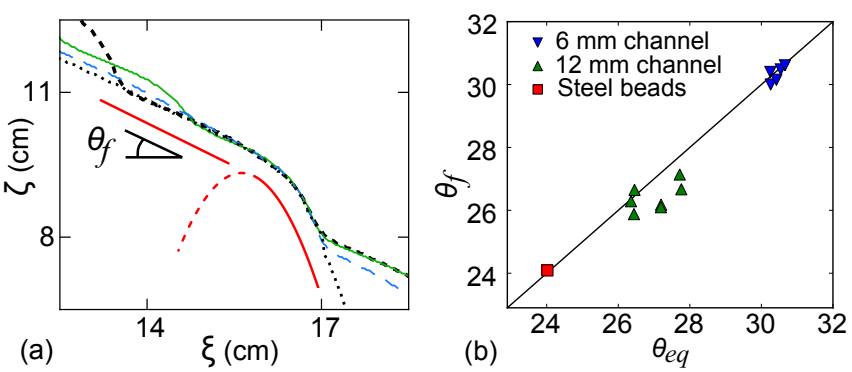

FIG. 2. (a) Superimposition of steps from a given experiment (black and blue dashed lines, solid green), and of a bigger step (dotted black), built from a higher initial slope angle: $43.7^{\circ}$. The shifted red lines are a fitting curve composed of a straight line of slope $31^{\circ}$ followed by a parabolic curve corresponding to a free fall. (b) Angle of the heap at the end of the experiment $\theta_{f}$, versus the steady angle of flow of the same granular material down a dry heap $\theta_{e q}$. Different flow rates are used to modify $\theta_{e q}$, and the cohesive heap is made of glass beads.

sidewall friction $[23,24]$ or captured by empirical laws close to the flow threshold [25]). Moreover, recent experiments have shown that the erosion of a wet granular surface results from a stochastic phenomenon [7]. These remarks rule out any intrinsic erosion threshold due to the wet pile. Therefore we measured the final angle $\theta_{f}$ for different flow rates, in channels of different widths, using stainless steel and glass beads, while keeping the same erodible heap (wet glass beads). Concurrently to each experiment, we directly measured the flow slope in the very same conditions, injecting the same flow rate in an initially empty cell (without the wet pile). In this well-known granular-flow geometry on a dry heap (called heap flow), the flowing layer stabilizes over a static dry heap at a given angle depending on the flow rate [23, 24]. Figure $2 \mathrm{~b}$ shows that this heap-flow angle $\theta_{e q}$ is equal to the final erosion angle, which proves that the erosion limit depends only on the flow properties. If the interface angle became smaller than the heap-flow angle, then a static dry layer would form between the wet grains and the flow preventing any further erosion process to occur. 
This criterion thus explains the angle of the back of the steps. One last consequence of the localization of the erosion on the step fronts is the reduction of the average erosion rate up to a factor 2 (see Suppl. Mat. [26]).

As already mentioned, the erosion of the cohesive heap does not always lead to the generation of steps. We explored under which conditions the interface remains flat. Due to the analogy with the shape and the propagation of the structures led by the pool and step instabilities in riverbeds [3], we expect to observe an inertial destabilization if the physical origin is identical. The underlying mechanism would be the phase lag between the topography and the local erosion rate due to inertia. Such phase lag exists for anti-dune or step-pool formations between the transport coefficient or the erosion and the shape of the interface [3, 4][27]. We expect therefore a stability criterion based on the Froude number $\mathrm{Fr}=u / \sqrt{g h \cos \theta_{i}}$, which compares the inertia with the gravity effects, where $g \cos \theta_{i}$ is the projection of the gravity perpendicular to the slope. We first check the relevance of a critical Froude number by plotting the different states observed in the two channels in the $\mathrm{Fr}-\theta_{i}$ diagram. As shown by the gray strip in the figure $3 \mathrm{a}$, our measurements show a critical Froude number, around 0.75 , above which the instability develops. The mechanism is thus the delay of the local erosion rate with respect to the topography induced by the flow inertia.

Consequently, in the parameter space $\left(\theta_{i}\right.$, flow rate) plotted in figure $3 \mathrm{~b}$, we can now draw the limits of the stable domain using iso-Fr lines. To compute the coordinates of these lines, we use the now established rheology for such dense dry granular flows $\mu(I)$ [28], which links the internal friction coefficient to the inertial number. More precisely, assuming the flat interface to be rigid at the time scale of the flow, we follow the approach proposed by Jop et al. [24] to compute the vertical velocity profile: we integrate numerically the velocity profile of a steady $2 \mathrm{D}$ granular flow on a rigid rough plane with a noslip boundary condition, and including friction at sidewalls [26]. After adjusting the parameters of the model on other dry heap experiments, we are able to draw the limits in the phase diagram (Fig. 3b). The solid line corresponds to the equilibrium angle of the flow over a dry heap. The dashed line is an iso-Froude curve $(\mathrm{Fr}=0.87$, see later) which delimit the stable region.

To further understand the mechanism of the instability, we model the flow using a Saint-Venant approach in a long-wave approximation [29](see Suppl. Mat. [26]). In the following, the influence of sidewalls will only be taken into account in the shape of the vertical velocity profile in the basic state. We discard this frictional term in the equations for sake of simplicity and because $h / W<0.48$ in the experiments [24]. The reference framework is the flat initial interface (the $x-z$ axes in fig. 1a) eroded at constant rate $\left\langle d \eta / d t>=-E_{0}\right.$, which represents the normal velocity of the mean interface. The evolution of the thickness $h$, the elevation of the interface $\eta$ and the mean velocity $u$ are then described by three equations: the mass conservation inside the flow of constant volume fraction and the momentum equation, both integrated over the depth $z$, and the erosion law. We verified the scale separation between the characteristic times of the slow erosion process (few $\mathrm{cm} / \mathrm{hr}$ ) and of the flow $(10 \mathrm{~cm} / \mathrm{s})$. This quasistatic evolution allows to neglect the mass flux into the flow due to erosion and the time derivative in the momentum equation:

$$
\rho \frac{\partial\left(\alpha h u^{2}\right)}{\partial x}=\rho g h \cos \theta\left(\tan \theta-\mu_{b}(u, h)-\frac{\partial h}{\partial x}\right),
$$

where $\alpha$ takes into account the vertical velocity profile (e.g. 5/4 for a Bagnold velocity profile or $4 / 3$ for a linear velocity profile as in our case). The left term is the inertia, and in the right hand side, from left to right, the terms are the weight of the layer, the basal friction and the derivative of the pressure. The basal friction is modeled by the $\mu(I)$ rheology [12, 28]. Following recent results on granular erosion [7], the erosion rate $\frac{\partial \eta}{\partial t}$ is proportional to $-\frac{F_{\text {cap }}}{F} \exp \left(-\frac{F_{\text {cap }}}{F}\right)$, where $F_{\text {cap }}$ is the capillary force responsible for the cohesion and $F$ is the mean force acting on grains. We used forces instead of stresses because the model was developed at the grain scale. In addition, we focus here only on the physical mechanism of the instability not on the influence of the cohesion on erosion [7]. As shown by the Fig. 3a,b, the erosion instability is linked to the inertia of the flowing grains, so $F$ must depend on their inertia. A recent numerical study [19] proposes that the average force of impacting grains is linked to their kinetic energy. We thus assume that $F$ is proportional to $\left(\rho u^{2}\right)^{\beta / 2}$ with $\beta=1.2$ [19]. The parameters are made dimensionless using the steady state erosion rate of the flat interface $E_{0}$, the thickness $h_{0}$, the flow rate per unit width $q_{0}$, and the mean force $F_{0}$ : $\tilde{h}=h / h_{0}, \tilde{x}=x / h_{0}, \tilde{\eta}=\eta / h_{0}, \tilde{u}=u h_{0} / q_{0}, \tilde{t}=t E_{0} / h_{0}$. After linearization around the initial steady state on a flat interface of slope $\theta_{i}$ we decompose the flow into normal modes $\propto \exp -i(\tilde{k} \tilde{x}+\tilde{\omega} \tilde{t})$ and find the growth rate:

$$
\tilde{\sigma}=\Im(\tilde{\omega})=c \frac{\tilde{k}^{2}\left(\alpha \mathrm{Fr}^{2}-1\right)}{(a-b)^{2}+\tilde{k}^{2}\left(\alpha \mathrm{Fr}^{2}-1\right)^{2}},
$$

where $a$ and $b$ are $\partial \mu_{b} / \partial u$ and $\partial \mu_{b} / \partial h$ [12][26], and $c=$ $\beta\left(F_{\text {cap }} / F_{0}-1\right)$. A critical Froude number is found above which the interface is unstable: $\operatorname{Fr}_{c}=\alpha^{-1 / 2}=0.87$ for $\alpha=4 / 3$. We use this value to plot the iso-Froude curve in figure $3 \mathrm{~b}$ showing a good agreement with the experimental results. Moreover, we can compare the predicted initial growth rate to the experimental ones, measuring the height of the steps in the early exponential regime. Figure 3c shows the measured growth rates of the initial steps rising from the flat interface for different experiments. The most unstable mode is obtained 

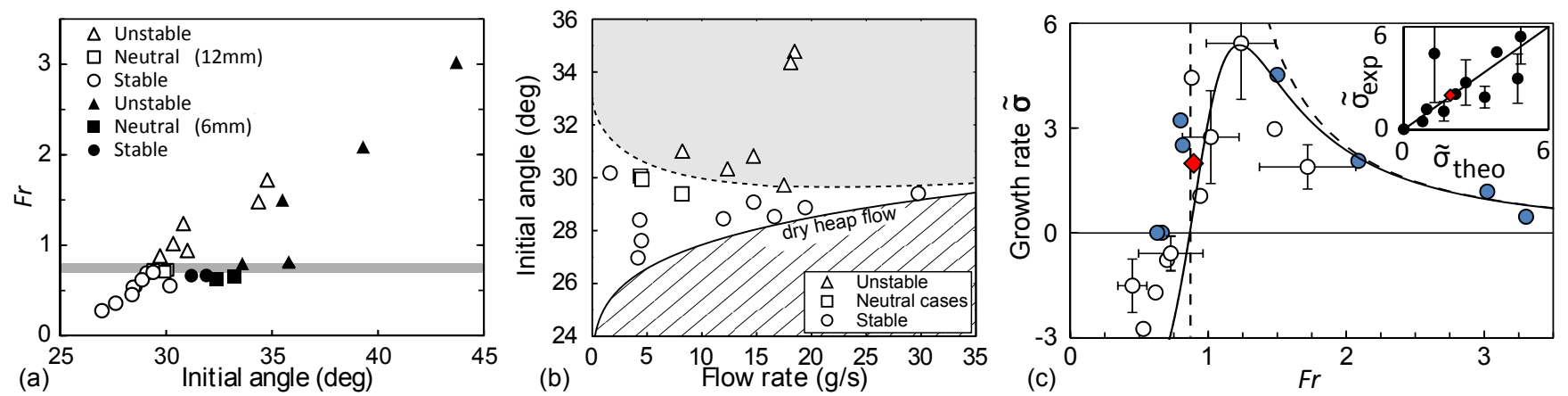

FIG. 3. (a) Evolution of the interface with the initial Fr. The symbols depict the evolution of the interface. The borders of the gray strip are 0.7 and 0.8 . (b) $\left(\theta_{i}\right.$-flow rate) phase diagram in the $12 \mathrm{~mm}$-wide channel. The solid line is the heap flow angle $\theta_{e q}$, under which erosion is not possible. The dashed line is the curve $\mathrm{Fr}=0.87$. (c) Dimensionless growth rates of the most unstable mode as a function of the Froude number in experiments for glass beads in the 6 mm channels $(\bullet)$ and 12 mm channel $(\circ)$ and steel beads in $12 \mathrm{~mm}$ channel $(\diamond)$. The curves correspond to a prediction of the theoretical growth rate (eq. (2)) for the most unstable mode $\tilde{k}=\infty$ (dashed line) and for a wave number in agreement with the experiment $h_{0} k=0.1$ (solid line) at an inclination of $\theta_{i}=30^{\circ}$. Inset: Direct comparison of growth rates using the measured wave numbers $\tilde{k}$ as a parameter.

with $\tilde{k}=\infty$ whose growth rate diverges at the threshold (dashed line). However, the model predictions for $k h_{0}=0.1$ and $\theta_{i}=30^{\circ}$ are in good agreement with the experimental data considering the error bars, noting that the experimental $\tilde{k}$ is not strictly constant but close to 0.1. The inset of Fig. 3c shows the comparison when using the experimental $\tilde{k}$. We checked that choosing a different shape factor $\alpha$ for the steady-state velocity profile does not change qualitatively the graph. Our study indicate that inertia drives this instability. Whenever the granular flow is supercritical $\left(\mathrm{Fr}>\mathrm{Fr}_{c}\right)$, the interface does not remain flat. This result is important to model correctly the evolution of the interface: if the mean erosion-force acting on the interface was linked to the weight of the layer or to the shear stress, the instability would not be predicted. Our findings strongly support the role of the impact of individual grains [19] in granular erosion mechanisms.

In conclusion, we have studied a new erosion instability occurring with granular flows over a cohesive granular bed. Above a critical Froude number, the interface is unstable and the shape of the steps is governed by the properties of the granular flows. We model with success the mechanisms in the framework of a Saint-Venant approach, extending the relevance of the depth-averaged methods to model complex evolutions of granular flows $[29,30]$. However, this model cannot predict any selection of a finite wavelength, additional longitudinal dissipation term in the momentum equation may be able to reduce the growth rate of too short wavelengths $[29,30]$. We finally identify that the inertia of grains governs the erosion mechanisms in dense granular flows. Further work is required to model the dilute erosion mechanisms in the fully developed state. We believe that our results could lead to a better understanding of geophysical flows.

The authors would like to acknowledge B. Andreotti who pointed out the cyclic step analogy and to thank E. Dressaire for her help with proofreading.

* pierre.jop@saint-gobain.com; http://svi.cnrs.fr

[1] D. R. Montgomery and W. E. Dietrich, Water Resour. Res. 25, 1907 (1989).

[2] A. Vianello and V. D'Agostino, Geomorphology 83, 266 (2007).

[3] G. Parker and N. Izumi, J. Fluid Mech. 419, 203 (2000).

[4] B. Andreotti, P. Claudin, O. Devauchelle, O. Durán, and A. Fourrière, J. Fluid Mech. 690, 94 (2012).

[5] A. Mangeney, O. Roche, O. Hungr, N. Mangold, G. Faccanoni, and A. Lucas, J. Geophys. Res. 115, F03040 (2010).

[6] R. M. Iverson, J. Geophys. Res. 117, F03006 (2012).

[7] G. Lefebvre and P. Jop, Phys. Rev. E 88, 032205 (2013).

[8] A. S. McEwen, C. J. Hansen, W. A. Delamere, E. M. Eliason, K. E. Herkenhoff, L. Keszthelyi, V. C. Gulick, R. L. Kirk, M. T. Mellon, and J. A. Grant et al., Science 317, 1706 (2007).

[9] J. D. Pelletier, K. J. Kolb, A. S. McEwen, and R. L. Kirk, Geology 36, 211 (2008).

[10] D. R. Montgomery, J. L. Bandfield, and S. K. Becker, J. Geophys. Res. 117, E03005 (2012).

[11] F. Charru, B. Andreotti, and P. Claudin, Annu. Rev. Fluid Mech. 45, 469 (2013).

[12] Y. Forterre and O. Pouliquen, J. Fluid Mech. 486, 21 (2003).

[13] N. Taberlet, P. Richard, E. Henry, and R. Delannay, Europhys. Lett. 68, 515 (2004).

[14] F. Malloggi, J. Lanuza, B. Andreotti, and E. Clément, Europhys. Lett. 75, 825 (2006).

[15] E. Martínez, C. Pérez-Penichet, O. Sotolongo-Costa, O. Ramos, K. Måløy, S. Douady, and E. Altshuler, Phys. Rev. E 75, 031303 (2007).

[16] A.-F. Bitbol, N. Taberlet, S. Morris, and J. McElwaine, Phys. Rev. E 79, 1 (2009).

[17] I. J. Hewitt, N. J. Balmforth, and J. N. McElwaine, J. 
Fluid Mech. 692, 446 (2012).

[18] L. Hsu, W. E. Dietrich, and L. S. Sklar, J. Geophys. Res. 113 (2008).

[19] B. Yohannes, L. Hsu, W. E. Dietrich, and K. M. Hill, J. Geophys. Res. 117, F02027 (2012).

[20] M. Scheel, R. Seemann, M. Brinkmann, M. di Michiel, A. Sheppard, B. Breidenbach, and S. Herminghaus, Nature Mat. 7, 189 (2008).

[21] S. J. Bennett, S. N. Alonso, Carlos V.and Prasad, and M. J. M. Römkens, Water Resour. Res. 36, 1911 (2000).

[22] O. Pouliquen, Phys. Fluids 11, 542 (1999).

[23] N. Taberlet, P. Richard, A. Valance, W. Losert, J. Pasini, J. T. Jenkins, and R. Delannay, Phys. Rev. Lett. 91, 264301 (2003).
[24] P. Jop, Y. Forterre, and O. Pouliquen, J. Fluid Mech. 541, 167 (2005).

[25] S. Deboeuf, E. Lajeunesse, O. Dauchot, and B. Andreotti, Phys. Rev. Lett. 97, 158303 (2006).

[26] see Supplemental material at [URL will be inserted by publisher] for details of the model.

[27] This analogy holds only if the erosion (or transport) process is driven by the inertia of the flow: The shear stress is the link between erosion and inertia in water flows. [3].

[28] GdR MiDi, Eur. Phys. J. E 14, 341 (2004).

[29] Y. Forterre, J. Fluid Mech. 563, 123 (2006).

[30] J. M. N. T. Gray and A. N. Edwards, J. Fluid Mech. 755, 503 (2014). 